\title{
EMBEDDING THE BRAID GROUP IN MAPPING CLASS GROUPS
}

\author{
BŁażej Szepietowski
}

\begin{abstract}
Motivated by a question of B. Wajnryb we construct embeddings of the braid group in mapping class groups of surfaces, which are not geometric in the sense that the images of standard generators are not Dehn twists. Our construction uses non-orientable surfaces and the fact that the mapping class group of such a surface embeds via lifting of homeomorphisms in the mapping class group of its orientable double cover.
\end{abstract}

\section{Introduction}

Let $S$ be a compact connected surface, possibly with boundary and with a finite set $P$ of distinguished points in the interior of $S$ called punctures. The mapping class group $\mathcal{M}(S, P)$ is the group of isotopy classes of all homeomorphisms $S \rightarrow S$ which are equal to the identity on the boundary $\partial S$, preserve the set $P$, and preserve orientation if $S$ is orientable. The isotopies are required to fix the points of $\partial S$ and the punctures. If $P$ is empty, then we abbreviate the notation to $\mathcal{M}(S)$.

The braid group on $n$ strands, denoted by $B_{n}$, may be defined by the presentation with generators $\sigma_{1}, \ldots, \sigma_{n-1}$ and relations:

$$
\begin{aligned}
\sigma_{i} \sigma_{j} & =\sigma_{j} \sigma_{i} & & \text { if }|i-j| \geq 2, \\
\sigma_{i} \sigma_{i+1} \sigma_{i} & =\sigma_{i+1} \sigma_{i} \sigma_{i+1} & & \text { for } i=1, \ldots, n-2 .
\end{aligned}
$$

Over the years, more and more connections have been discovered between the mapping class group and the braid group, which share many very specific properties. Actually, $B_{n}$ is isomorphic to the mapping class group of a disc with $n$ punctures via an isomorphism which sends the generators $\sigma_{i}$ to half-twists about arcs connecting pairs of punctures (see

2000 Mathematics Subject Classification. Primary: 20F36; Secondary: 57N05, 20 F38. Key words. Mapping class group, braid group, non-geometric embedding. Supported by MNiSW grant N N201 366436. 
Section 3 for details). This fact is so convenient that it is often used as a definition of the braid group.

The braid group can also be seen as a subgroup of the mapping class group a surface without punctures in the following way. Suppose that we are given $n-1$ two-sided simple closed curves $\alpha_{1}, \ldots, \alpha_{n-1}$ on a surface $S$, such that $\alpha_{i}$ and $\alpha_{j}$ are disjoint whenever $|i-j| \geq 2$, and $\alpha_{i}$ intersects $\alpha_{i+1}$ transversally at one point for $i=1, \ldots, n-2$. Let $\Sigma \subseteq S$ be a closed regular neighborhood of the union of the curves $\alpha_{i}$. Fix an orientation of $\Sigma$ and let $t_{\alpha_{i}}$ denote the right Dehn twist about $\alpha_{i}$. Then it follows from the presentation of $B_{n}$ and well known properties of Dehn twists, that the assignment $\sigma_{i} \mapsto t_{\alpha_{i}}$ for $i=1, \ldots, n-1$ extends to a homomorphism $B_{n} \rightarrow \mathcal{M}(S)$. If $\Sigma=S$ then this homomorphism is injective by a theorem of Birman and Hilden (see [4] and [5]).

It is natural to ask if one can see the braid group as a subgroup of the mapping class group in some other way. The following definition and question are due to Wajnryb $[\mathbf{1 2}]$.

Definition 1. A homomorphism form the braid group to the mapping class group, which maps the generators $\sigma_{i}$ on Dehn twists is called geometric.

Question 2. Are there non-geometric embeddings of the braid group in the mapping class group?

The aim of this paper is to give an affirmative answer to this question by showing how such embeddings can be constructed using nonorientable surfaces. In Section 2 we show that the mapping class group of a non-orientable surface $N$ is isomorphic to a subgroup of the mapping class group of its orientable double cover $S$ containing no (powers of) Dehn twists. Thus, from every embedding $\varphi: B_{n} \rightarrow \mathcal{M}(N)$ we can obtain an embedding $\psi: B_{n} \rightarrow \mathcal{M}(S)$, whose image contains no Dehn twists. In Section 3 we use the fact that the braid group is isomorphic to the mapping class group of a punctured disc, to construct an embedding $\varphi: B_{n} \rightarrow \mathcal{M}(N)$, where $N$ is obtained from the disc by replacing each puncture by the Möbius strip. In Section 4 we describe the image $\psi\left(\sigma_{i}\right)$ of a standard generator of the braid group, where $\psi: B_{n} \rightarrow \mathcal{M}(S)$ is the embedding obtained by "lifting" $\varphi$ to the mapping class group of the orientable double cover of $N$.

\section{Preliminaries}

Suppose that $F \subseteq S$ is a subsurface such that $\partial F \cap P=\emptyset$. Every homeomorphism of $F$ equal to the identity on $\partial F$ may be extended by the 
identity on $S \backslash F$ to a homeomorphism of $S$. Thus we have the induced homomorphism

$$
i_{*}: \mathcal{M}(F, P \cap F) \rightarrow \mathcal{M}(S, P),
$$

whose kernel is described in Theorem 4.1 of [9] and Theorem 3.6 of [10].

Let $N$ be a non-orientable surface of genus $g$ with $b$ boundary components and no punctures. Let $p: S \rightarrow N$ be the orientation double covering of $N$, so that $S$ is an orientable surface of genus $g-1$ with $2 b$ boundary components, and let $\tau: S \rightarrow S$ be the covering involution. We call a homeomorphism $h: S \rightarrow S$ symmetric if $h \tau=\tau h$. If $h$ is symmetric, then it projects to $h^{\prime}: N \rightarrow N$ defined by $p h=h^{\prime} p$. We say that $h$ is a lift of $h^{\prime}$. Let $\mathcal{S} \mathcal{M}(S)$ denote the subgroup of $\mathcal{M}(S)$ consisting of isotopy classes of symmetric homeomorphisms which preserve orientation and are equal to the identity on $\partial S$.

Lemma 3. Suppose that $g+2 b \geq 3$. Then $\mathcal{M}(N)$ and $\mathcal{S} \mathcal{M}(S)$ are isomorphic via $\pi: \mathcal{M}(N) \rightarrow \mathcal{S} \mathcal{M}(S)$ defined as $\pi(h)=\tilde{h}$, where $\tilde{h}$ is the unique orientation preserving lift of $h$.

Proof: Suppose that $N$ is closed. Let $\mathcal{M}^{\diamond}(S)$ denote the extended mapping class group of $S$ including also the classes of homeomorphisms reversing orientation, and let $\mathcal{S M}^{\diamond}(S)$ denote its subgroup consisting of classes of symmetric homeomorphisms. By [3], projecting symmetric homeomorphisms induces surjective homomorphism $\mathcal{S M}^{\diamond}(S) \rightarrow \mathcal{M}(N)$ with kernel $\langle\tau\rangle$. Since the involution $\tau$ reverses orientation, $\mathcal{S M}^{\diamond}(S) /\langle\tau\rangle$ is obviously isomorphic to $\mathcal{S} \mathcal{M}(S)$. The lemma follows.

If $N$ is not closed, then we obtain a closed non-orientable surface $N^{\prime}$ of genus $g^{\prime}=g+2 b$ by gluing to $N$ one copy of a torus with one hole along each of the $b$ boundary components. Let $p^{\prime}: S^{\prime} \rightarrow N^{\prime}$ be an orientation double covering, such that $S=p^{-1}(N)$ and $\left.p^{\prime}\right|_{S}=p$. If $T$ is a connected component of $N^{\prime} \backslash \operatorname{int}(N)$ then the pre-image $\widetilde{T}=p^{-1}(T)$ is not connected. That is because if there was a path in $\widetilde{T}$ connecting any point $x$ to $\tau(x)$, then its projection would be a one-sided closed path in $T$, because $\tau$ is orientation reversing, which is impossible. Since $T$ has one boundary component and $\chi(T)=-1, \widetilde{T}$ has two boundary components and $\chi(\widetilde{T})=-2$. It follows that $\widetilde{T}$ is the disjoint union of two copies of a torus with one hole. Hence $S^{\prime} \backslash S$ is the disjoint union of $2 b$ such copies. By Corollary 3.8 in [10] and Corollary 4.2 in [9] the induced homomorphisms $i_{*}: \mathcal{M}(N) \rightarrow \mathcal{M}\left(N^{\prime}\right)$ and $i_{*}: \mathcal{M}(S) \rightarrow \mathcal{M}\left(S^{\prime}\right)$ are injective. From the previous argument we have the isomorphism $\pi: \mathcal{M}\left(N^{\prime}\right) \rightarrow \mathcal{S} \mathcal{M}\left(S^{\prime}\right)$. We want to show that $\pi\left(i_{*}(\mathcal{M}(N))\right)=i_{*}(\mathcal{S} \mathcal{M}(S))$. 
If $h$ is a symmetric homeomorphism of $S$ equal to the identity on $\partial S$, then its extension to $S^{\prime}$ is also symmetric and it projects to a homeomorphism of $N^{\prime}$ equal to the identity on $N^{\prime} \backslash N$. Hence $i_{*}(\mathcal{S} \mathcal{M}(S)) \subseteq$ $\pi\left(i_{*}(\mathcal{M}(N))\right)$.

Suppose that $f$ is a homeomorphism of $N^{\prime}$ equal to the identity on $N^{\prime} \backslash N$ and let $\tilde{f}$ be the orientation preserving lift of $f$. We need to show that $\tilde{f}$ is equal to the identity on $S^{\prime} \backslash S$. It suffices to prove that $\tilde{f}$ fixes every component of $\partial S$. If $\gamma$ is a connected component of $\partial S$, then either $\tilde{f}(\gamma)=\gamma$ or $\tilde{f}(\gamma)=\tau(\gamma)$. In the latter case we have $\tau \tilde{f}(\gamma)=\gamma$, and since $\tau \tilde{f}$ preserves $S$ and reverses its orientation, it must reverse orientation of $\gamma$. Thus the projection of $\tau \tilde{f}$ reverses orientation of $p(\gamma)$. But $\tau \tilde{f}$ projects to $f$, which is equal to identity on $p(\gamma)$. Hence $\tilde{f}(\gamma)=\gamma$ and $\pi\left(i_{*}(\mathcal{M}(N))\right) \subseteq i_{*}(\mathcal{S M}(S))$

The following lemma asserts that $\mathcal{S M}(S)$ contains no nontrivial powers of Dehn twists.

Lemma 4. Suppose that $\gamma$ is a simple closed curve in $S$ which is not nullhomotopic and let $t_{\gamma}$ denote the right Dehn twist about $\gamma$. If $t_{\gamma}^{i} \in \mathcal{S} \mathcal{M}(S)$ for some integer $i$, then $i=0$.

Proof: If $t_{\gamma}^{i} \in \mathcal{S M}(S)$ then $\tau t_{\gamma}^{i} \tau=t_{\gamma}^{i}$. On the other hand, since $\tau$ is orientation reversing, $\tau t_{\gamma}^{i} \tau=t_{\tau(\gamma)}^{-i}$. Thus $t_{\gamma}^{i}=t_{\tau(\gamma)}^{-i}$, which implies, by the well known property of Dehn twists (see for example Theorem 4.1 in $[6]$ ), that $\gamma$ is isotopic to $\tau(\gamma)$ and $i=-i$. Hence $i=0$.

We record the following immediate corollary, although it is not used in this paper.

Corollary 5. The index of $\mathcal{S} \mathcal{M}(S)$ in $\mathcal{M}(S)$ is infinite.

Proof: Let $\gamma$ be any simple closed curve in $S$ which is not null-homotopic. By Lemma 4 different powers of $t_{\gamma}$ represent different cosets of $\mathcal{S M}(S)$ in $\mathcal{M}(S)$.

From Lemmas 3 and 4 we obtain the following theorem.

Theorem 6. Suppose that $\varphi: B_{n} \rightarrow \mathcal{M}(N)$ is any embedding of the braid group in the mapping class group of a non-orientable surface of genus $g$ with $b$ boundary components, where $g+2 b \geq 3$. Define $\psi$ to be the composition $\pi \circ \varphi$, where $\pi: \mathcal{M}(N) \rightarrow \mathcal{S} \mathcal{M}(S)$ is the isomorphism from Lemma 3. Then $\psi$ is an embedding of $B_{n}$ in the mapping class group of an orientable surface of genus $g-1$ with $2 b$ boundary components, whose image contains no nontrivial powers of Dehn twists. In particular $\psi$ is not geometric. 
Suppose that $\varphi: B_{n} \rightarrow \mathcal{M}(N)$ in Theorem 6 is geometric, so that $\varphi\left(\sigma_{i}\right)=t_{\gamma_{i}}$ for $i=1, \ldots, n-1$, where $\gamma_{i}$ is a two-sided simple close curve in $N$. The pre-image $p^{-1}\left(\gamma_{i}\right)$ consists of two disjoint simple closed curves $\gamma_{i}^{\prime}, \gamma_{i}^{\prime \prime}$. If $t_{\gamma_{i}^{\prime}}$ and $t_{\gamma_{i}^{\prime \prime}}$ are right Dehn twists, then since $\tau$ is orientation reversing, we have $\tau t_{\gamma_{i}^{\prime}} \tau=t_{\gamma_{i}^{\prime \prime}}^{-1}$. Since twists about $\gamma_{i}^{\prime}$ and $\gamma_{i}^{\prime \prime}$ commute, it follows that $t_{\gamma_{i}^{\prime}} t_{\gamma_{i}^{\prime \prime}}^{-1}$ is symmetric. Its projection is clearly a Dehn twist about $\gamma_{i}$. Thus $\psi\left(\sigma_{i}\right)=\pi\left(t_{\gamma_{i}}\right)=\left(t_{\gamma_{i}^{\prime}} t_{\gamma_{i}^{\prime \prime}}^{-1}\right)^{ \pm 1}$ and the embedding $\psi: B_{n} \rightarrow \mathcal{M}(S)$ can be seen as the composition $\psi=\psi^{\prime} \circ \rho$, where $\rho: B_{n} \rightarrow B_{2 n}$ is the doubling homomorphism defined as $\rho\left(\sigma_{i}\right)=\sigma_{i} \sigma_{2 n-i}^{-1}$ for $i=1, \ldots, n-1$, and $\psi^{\prime}: B_{2 n} \rightarrow \mathcal{M}(S)$ is a geometric embedding. In order to construct a more interesting embedding $\psi: B_{n} \rightarrow \mathcal{M}(S)$, we have to start from an embedding $\varphi: B_{n} \rightarrow \mathcal{M}(N)$ which is not geometric.

\section{Non-orientable surface}

The aim of this section is to construct an embedding $\varphi: B_{n} \rightarrow \mathcal{M}(N)$ of the braid group in the mapping class group of a non-orientable surface, which is not geometric in the sense of Definition 1.

Let $D$ be a disc with $n$ punctures $P=\left\{x_{1}, \ldots, x_{n}\right\}$. For $i=1, \ldots, n$ let $U_{i}$ be a small closed disc in $D$ containing $x_{i}$ and disjoint from $\partial D$. We assume that $U_{i} \cap U_{j}=\emptyset$ for $i \neq j$. Denote by $\alpha_{i}$ the boundary of $U_{i}$. Define $F$ to be $D$ with the union of interiors of $U_{i}$ deleted. Fix an orientation of $F$ and orient each $\alpha_{i}$ as boundary curve of $F$. Let $M$ be the Möbius strip. We construct a non-orientable surface $N$ by gluing a copy of $M$ to $\partial F$ along $\alpha_{i}$ regarded as a function $\alpha_{i}: \partial M \rightarrow \partial F$ for $i=1, \ldots, n$.

Suppose that $h \in \mathcal{M}(D, P)$. Then there is a permutation $\theta \in \Sigma_{n}$ such that $h\left(x_{i}\right)=x_{\theta(i)}$ for $i=1, \ldots, n$. In the isotopy class $h$ there is a homeomorphism, which we also denote by $h$, such that $h\left(U_{i}\right)=U_{\theta(i)}$ and $h \circ \alpha_{i}=\alpha_{\theta(i)}$ as functions, for $i=1, \ldots, n$. The restriction $\left.h\right|_{F}$ may be extended to a homeomorphism $h^{\prime}: N \rightarrow N$ by the identity between each pair of copies of $M$ glued to $\partial F$ along $\alpha_{i}$ and $\alpha_{\theta(i)}$.

Theorem 7. The map $\eta: \mathcal{M}(D, P) \rightarrow \mathcal{M}(N)$ defined as $\eta(h)=h^{\prime}$ is a well defined injective homomorphism.

Proof: To prove that $\eta$ is well defined, suppose that $h$ and $g$ are two homeomorphisms of $D$ representing the same element of $\mathcal{M}(D, P)$ and such that $h \circ \alpha_{i}=\alpha_{\theta(i)}=g \circ \alpha_{i}$ for $i=1, \ldots, n$. Then the restriction to $F$ of $h g^{-1}$ yields an element of the kernel of $i_{*}: \mathcal{M}(F) \rightarrow \mathcal{M}(D, P)$ induced by the inclusion of $F$ in $D$. It follows from Theorem 4.1 of [9] 
that $h$ and $g$ differ by some product of Dehn twists about the curves $\alpha_{i}$. Since a Dehn twist about a curve bounding a Möbius strip is trivial, the extensions $h^{\prime}$ and $g^{\prime}$ are isotopic on $N$.

For injectivity, suppose that $\eta(h)$ is isotopic to the identity on $N$. Then $h\left(x_{i}\right)=x_{i}$ and $h$ may be represented by a homeomorphism equal to the identity on $U_{i}$ for $i=1, \ldots, n$. The restriction $\left.h\right|_{F}$ yields an element of the kernel of $i_{*}: \mathcal{M}(F) \rightarrow \mathcal{M}(N)$ induced by the inclusion of $F$ in $N$. By Theorem 3.6 of [10], this kernel is generated by Dehn twists about the curves $\alpha_{i}$. It follows that $h$ is trivial in $\mathcal{M}(D, P)$.

In order to obtain an embedding $\alpha: B_{n} \rightarrow \mathcal{M}(N)$, we have to define an isomorphism $B_{n} \rightarrow \mathcal{M}(D, P)$. This can be done as follows. For $i=1, \ldots, n-1$ let $\beta_{i}$ be an embedded arc in $D$ joining $x_{i}$ and $x_{i+1}$ such that $\beta_{i} \cap P=\left\{x_{i}, x_{i+1}\right\}$. We assume that the interiors of $\beta_{i}$ and $\beta_{j}$ are disjoint for $i \neq j$. Let $D_{i}$ be a closed regular neighborhood of $\beta_{i}$, so that $D_{i}$ is a disc with two punctures. There is a homeomorphism $h_{\beta_{i}}: D \rightarrow D$, equal to the identity on the complement of the interior of $D_{i}$, which interchanges the punctures and such that $h_{\beta_{i}}^{2}$ is the Dehn twist about $\partial D_{i}$ right with respect to some fixed orientation of $D$ (Figure 1). Such homeomorphism is called half-twist about $\beta_{i}$. It is a classical result that the assignment $\sigma_{i} \mapsto h_{\beta_{i}}, i=1, \ldots, n-1$ extends to an isomorphism $B_{n} \rightarrow \mathcal{M}(D, P)$ (see $\left.[\mathbf{2}]\right)$.

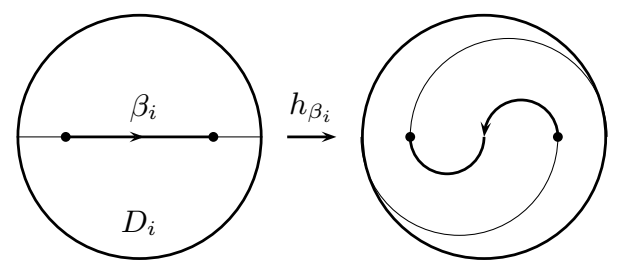

Figure 1. The half-twist $h_{\beta_{i}}$.

For $i=1, \ldots, n-1$ we define $u_{i} \in \mathcal{M}(N)$ as $u_{i}=\eta\left(h_{\beta_{i}}\right)$. Each $u_{i}$ is supported in a Klein bottle with a hole $K_{i}$ in Figure 2, where the shaded discs represent cross-caps, i.e. their interiors should be removed, and then antipodal points in each resulting boundary component should be identified. Let $\mu_{i}$ and $\mu_{i+1}$ be the cores of the Möbius strips glued to $F$ along $\alpha_{i}$ and $\alpha_{i+1}$ respectively. Observe that $\alpha_{i}$ is homotopic with the curve going twice along $\mu_{i}$. We have $u_{i}\left(\alpha_{i}\right)=\alpha_{i+1}$ and $u_{i}\left(\mu_{i}\right)=\mu_{i+1}\left(u_{i}\right.$ interchanges the two cross-caps). Let $t_{\gamma_{i}}$ denote the Dehn twist about 
the two-sided curve $\gamma_{i}$ in Figure 2 in the direction indicated by the arrows. The composition $t_{\gamma_{i}} u_{i}$ is isotopic to a cross-cap slide, or Y-homeomorphism, defined by Lickorish, which is not a product of Dehn twists (see $[\mathbf{8}]$ ). It follows that $u_{i}$ is not a product of Dehn twists (cf. [11]). From Theorem 7 we obtain the following corollary.

Corollary 8. The mapping $\varphi\left(\sigma_{i}\right)=u_{i}$ for $i=1, \ldots, n-1$ extends to an injective homomorphism $\varphi: B_{n} \rightarrow \mathcal{M}(N)$, where $N$ is a non-orientable surface of genus $n$ with one boundary component. Moreover, $\varphi$ is not geometric in the sense of Definition 1.

Note that $u_{i}^{2}=t_{\delta_{i}}$, where $t_{\delta_{i}}$ is the right Dehn twist about $\delta_{i}=\partial K_{i}$. One can think of $\varphi$ as a realization of the $m=2$ case of the first faithful Wada action described in [1, Historical Remarks 9.8]. The following lemma will be used in the next section.

Lemma 9. Suppose that $h \in \mathcal{M}\left(K_{i}\right)$ preserves the isotopy class of the curve $\alpha_{i}$, reverses its orientation, and $h^{2}=t_{\delta_{i}}$. Then $h=t_{\gamma_{i}} u_{i}$.

Proof: The group $\mathcal{M}\left(K_{i}\right)$ is generated by $u_{i}$ and $t_{\gamma_{i}}$. This follows from Theorem 4.9 of $[\mathbf{7}]$, see also [11]. These generators satisfy $u_{i} t_{\gamma_{i}} u_{i}^{-1}=$ $t_{\gamma_{i}}^{-1}$, thus $h=t_{\gamma_{i}}^{k} u_{i}^{e} t_{\delta_{i}}^{l}$, where $k, l$ are integers and $e$ is 1 or 0 . Recall that $H_{1}\left(K_{i}\right)$ is the free abelian group on generators $\left[\mu_{i}\right]$ and $\left[\mu_{i+1}\right]$, the homology classes of the curves $\mu_{i}$ and $\mu_{i+1}$. For appropriate choices of orientation we have: $u_{i}\left[\mu_{i}\right]=\left[\mu_{i+1}\right], u_{i}\left[\mu_{i+1}\right]=\left[\mu_{i}\right], t_{\gamma_{i}}\left[\mu_{i}\right]=2\left[\mu_{i}\right]+$ $\left[\mu_{i+1}\right], t_{\gamma_{i}}\left[\mu_{i+1}\right]=-\left[\mu_{i}\right]$. Also $\left[\alpha_{i}\right]=2\left[\mu_{i}\right]$ and it is easy to check that $h\left[\alpha_{i}\right]=-\left[\alpha_{i}\right]$ implies $k=e=1$. Now $h^{2}=t_{\delta_{i}}^{2 l+1}$ and since $t_{\delta_{i}}$ has infinite order we have $l=0$.

\section{Orientable surface}
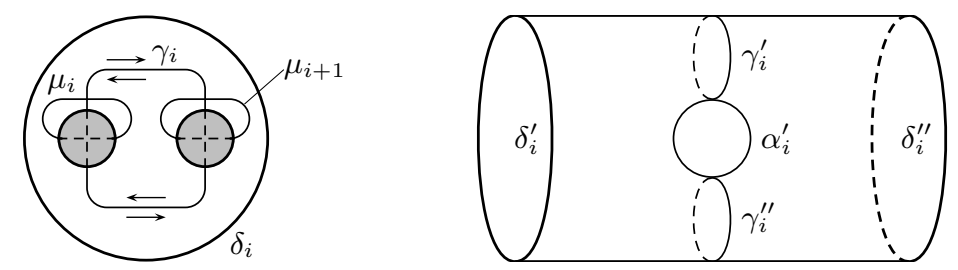

Figure 2. The surfaces $K_{i}$ (left) and $\widetilde{K_{i}}$ (right).

Let $p: S \rightarrow N$ be the orientation double cover, where $N$ is a nonorientable surface of genus $n$ with one boundary component. Thus $S$ is 
an orientable surface of genus $n-1$ with two boundary components. We define $\psi: B_{n} \rightarrow \mathcal{M}(S)$ to be the composition $\pi \circ \varphi$, where $\varphi: B_{n} \rightarrow$ $\mathcal{M}(N)$ is the embedding defined in the previous section. By Theorem 6 $\psi$ is a non-geometric embedding. The aim of this section is to describe the images $\psi\left(\sigma_{i}\right)$ of the generators of $B_{n}$.

Recall that the pre-image by $p$ of a one-sided curve in $N$ is a single curve in $S$, whereas the pre-image of a two-sided curve is a pair of disjoint curves. Let $K_{i} \subset N$ be the Klein bottle with one hole supporting the homeomorphism $u_{i}$ (Figure 2 left), and $\widetilde{K}_{i}=p^{-1}\left(K_{i}\right)$. Since $K_{i}$ has one boundary component and $\chi\left(K_{i}\right)=-1, \widetilde{K}_{i}$ has two boundary components and $\chi\left(\widetilde{K}_{i}\right)=-2$. In addition, $\widetilde{K}_{i}$ is connected and orientable so it is a torus with two holes. Define simple closed curves $\alpha_{i}^{\prime}, \gamma_{i}^{\prime}, \gamma_{i}^{\prime \prime}, \delta_{i}^{\prime}, \delta_{i}^{\prime \prime}$ on $S$ such that $p^{-1}\left(\mu_{i}\right)=\alpha_{i}^{\prime}, p^{-1}\left(\gamma_{i}\right)=\gamma_{i}^{\prime} \cup \gamma_{i}^{\prime \prime}, p^{-1}\left(\delta_{i}\right)=\delta_{i}^{\prime} \cup \delta_{i}^{\prime \prime}$ (Figure 2). We assume the convention that positive Dehn twists about the curves in Figure 2 are to the right. We have $\tau\left(\gamma_{i}^{\prime}\right)=\gamma_{i}^{\prime \prime}$ and $\tau$ reverses orientation of $S$, hence $\tau t_{\gamma_{i}^{\prime}} \tau=t_{\gamma_{i}^{\prime \prime}}^{-1}$. Since $\gamma_{i}^{\prime}$ and $\gamma_{i}^{\prime \prime}$ are disjoint, so the Dehn twists $t_{\gamma_{i}^{\prime}}$ and $t_{\gamma_{i}^{\prime \prime}}$ commute. It follows that $t_{\gamma_{i}^{\prime}} t_{\gamma_{i}^{\prime \prime}}^{-1}$ is symmetric. Its projection is clearly a Dehn twist about $\gamma_{i}$ and upon interchanging $\gamma_{i}^{\prime}$ and $\gamma_{i}^{\prime \prime}$, we can assume that this projection is $t_{\gamma_{i}}$ defined above. Analogously $t_{\delta_{i}^{\prime}} t_{\delta_{i}^{\prime \prime}}^{-1}$ is symmetric and we assume that it projects to $t_{\delta_{i}}$.

Theorem 10. Let $\psi: B_{n} \rightarrow \mathcal{M}(S)$ be the embedding defined as the composition $\pi \circ \varphi$, where $\varphi: B_{n} \rightarrow \mathcal{M}(N)$ is defined on the generators as $\varphi\left(\sigma_{i}\right)=u_{i}$ for $i=1, \ldots, n-1$. Then

$$
\psi\left(\sigma_{i}\right)=t_{\gamma_{i}^{\prime}}^{-1} t_{\gamma_{i}^{\prime \prime}}\left(t_{\alpha_{i}^{\prime}} t_{\gamma_{i}^{\prime}} t_{\gamma_{i}^{\prime \prime}}\right)^{2} t_{\delta_{i}^{\prime \prime}}^{-1}
$$

for $i=1, \ldots, n-1$.

Proof: Let $\tilde{y}=\left(t_{\alpha_{i}^{\prime}} t_{\gamma_{i}^{\prime}} t_{\gamma_{i}^{\prime \prime}}\right)^{2} t_{\delta_{i}^{\prime \prime}}^{-1}$. Such element appears in Lemma 1 of $[\mathbf{3}]$. By repeating the calculations in $[3]$ it can be checked that $\tilde{y}$ is symmetric and $\tilde{y}^{2}=t_{\delta_{i}^{\prime}} t_{\delta_{i}^{\prime \prime}}^{-1}$, so its projection $y \in \mathcal{M}\left(N^{\prime}\right)$ satisfies $y^{2}=t_{\delta_{i}}$. It can be checked that $\tilde{y}$ preserves the isotopy class of the curve $\alpha_{i}^{\prime}$ and reverses its orientation. Since $p\left(\alpha_{i}^{\prime}\right)$ is homotopic to $\alpha_{i}$, it follows that $y$ preserves $\alpha_{i}$ and reverses its orientation. By Lemma 9 we have $y=t_{\gamma_{i}} u_{i}$ and $\psi\left(\sigma_{i}\right)=$ $\pi\left(u_{i}\right)=\pi\left(t_{\gamma_{i}}^{-1} y\right)=t_{\gamma_{i}^{\prime}}^{-1} t_{\gamma_{i}^{\prime \prime}} \tilde{y}$ as desired. 

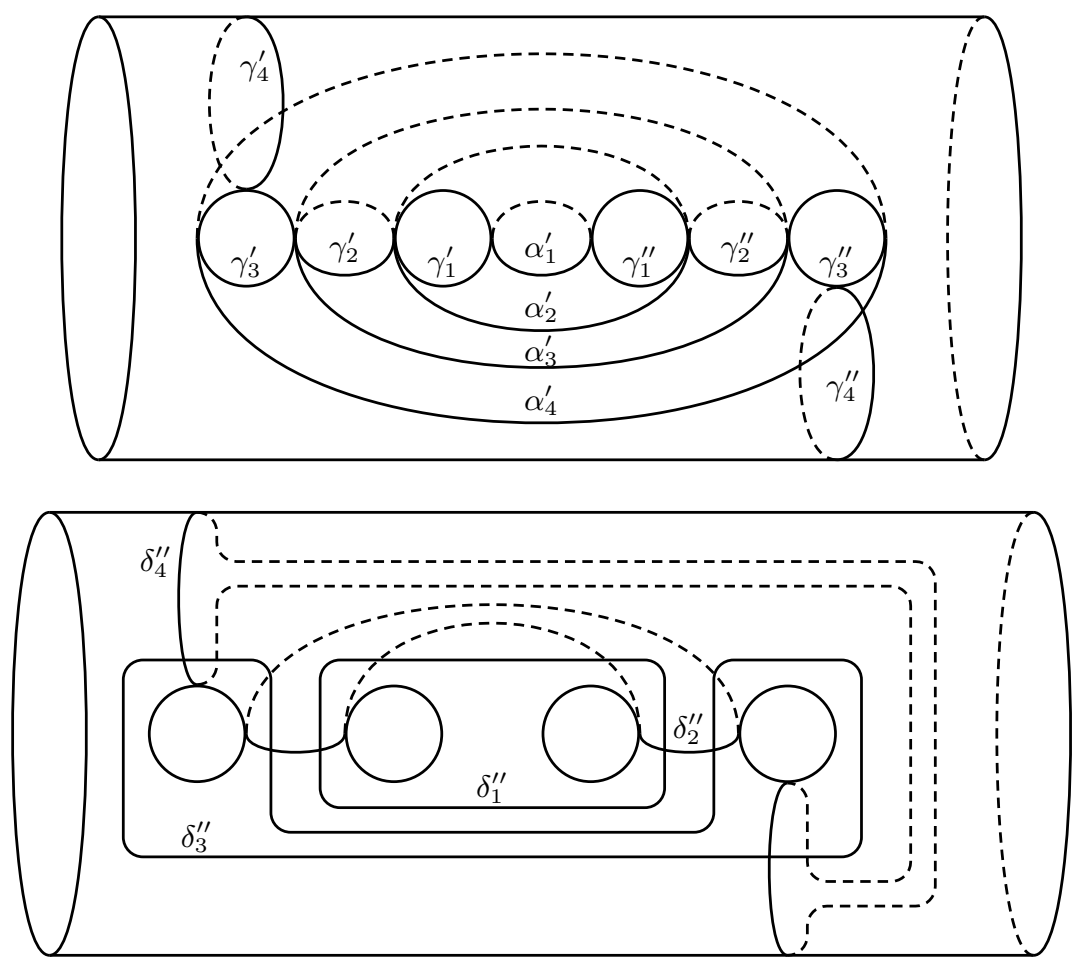

Figure 3. The configuration of curves in $S$.

Figure 3 shows the configuration of the curves involved in $\psi\left(\sigma_{i}\right)$ for $i=1, \ldots, 4$. If $x_{i}=\psi\left(\sigma_{i}\right)=t_{\gamma_{i}^{\prime}}^{-1} t_{\gamma_{i}^{\prime \prime}}\left(t_{\alpha_{i}^{\prime}} t_{\gamma_{i}^{\prime}} t_{\gamma_{i}^{\prime \prime}}\right)^{2} t_{\delta_{i}^{\prime \prime}}^{-1}$, then it may be checked that $x_{i+1} x_{i}$ maps $\alpha_{i+1}^{\prime}$ to $\alpha_{i}^{\prime}, \gamma_{i+1}^{\prime}$ to $\gamma_{i}^{\prime}, \gamma_{i+1}^{\prime \prime}$ to $\gamma_{i}^{\prime \prime}$ and $\delta_{i+1}^{\prime \prime}$ to $\delta_{i}^{\prime \prime}$. The resulting relations between Dehn twists give $x_{i+1} x_{i} x_{i+1}=x_{i} x_{i+1} x_{i}$.

Acknowledgement. A part of this paper was written during the author's stay at the Institute of Mathematics of the Polish Academy of Sciences.

\section{References}

[1] Ll. Bacardit And W. Dicks, Actions of the braid group, and new algebraic proofs of results of Dehornoy and Larue, Groups Complex. Cryptol. 1(1) (2009), 77-129. 
[2] J. S. Birman, "Braids, links, and mapping class groups", Annals of Mathematics Studies 82, Princeton University Press, Princeton, N.J.; University of Tokyo Press, Tokyo, 1974.

[3] J. S. Birman and D. R. J. Chillingworth, On the homeotopy group of a non-orientable surface, Proc. Cambridge Philos. Soc. $\mathbf{7 1}$ (1972), 437-448.

[4] J. S. Birman and H. M. Hilden, Lifting and projecting homeomorphisms, Arch. Math. (Basel) 23 (1972), 428-434.

[5] J. S. Birman and H. M. Hilden, On isotopies of homeomorphisms of Riemann surfaces, Ann. of Math. (2) 97 (1973), 424-439.

[6] N. V. Ivanov and J. D. McCarthy, On injective homomorphisms between Teichmüller modular groups. I, Invent. Math. 135(2) (1999), 425-486

[7] M. Korkmaz, Mapping class groups of nonorientable surfaces, Geom. Dedicata 89 (2002), 109-133.

[8] W. B. R. Lickorish, Homeomorphisms of non-orientable twomanifolds, Proc. Cambridge Philos. Soc. 59 (1963), 307-317.

[9] L. Paris and D. Rolfsen, Geometric subgroups of mapping class groups, J. Reine Angew. Math. 521 (2000), 47-83.

[10] M. Stukow, Commensurability of geometric subgroups of mapping class groups, Geom. Dedicata 143(1) (2009), 117-142.

[11] B. Szepietowski, The mapping class group of a nonorientable surface is generated by three elements and by four involutions, Geom. Dedicata 117 (2006), 1-9.

[12] B. WAJNRYB, Relations in the mapping class group, in: "Problems on mapping class groups and related topics", Proc. Sympos. Pure Math. 74, Amer. Math. Soc., Providence, RI, 2006, pp. 115-120.

Institute of Mathematics

Gdańsk University

Wita Stwosza 57

80-952 Gdańsk

Poland

E-mail address: blaszep@mat.ug.edu.pl

Primera versió rebuda el 4 de maig de 2009, darrera versió rebuda el 26 d'octubre de 2009. 